\title{
Efecto de los parámetros de soldadura sobre la microestructura de recargues nanoestructurados base hierro depositados por FCAW-S
}

\section{(Effect of welding parameters on microstructure of Fe-based Nanostructured Weld Overlay deposited through FCAW-S)}

\author{
Agustín Gualco ${ }^{1}$, Hernán G. Svoboda ${ }^{2,3}$, Estela S. Surian ${ }^{1}$ \\ ${ }^{1}$ Universidad Nacional de Lomas de Zamora, Facultad de Ingeniería, Secretaría de Investigación, Buenos Aires, Argentina. e-mail: \\ agustingualco@yahoo.com.ar \\ ${ }^{2}$ Universidad de Buenos Aires, Facultad de Ingeniería, Laboratorio de Materiales y Estructuras y Departamento de Ingeniería \\ Mecánica, Laboratorio de Materiales y Estructuras, INTECIN, Argentina. \\ ${ }^{3}$ Consejo Nacional de Investigaciones Cientificas y Técnicas, Argentina.
}

\section{Resumen}

Recientemente se han fabricado modernos alambres tubulares utilizados con el proceso de soldadura semiautomática bajo protección gaseosa y sin ella, que depositan aleaciones base Fe nanoestructurados, con alta resistencia al desgaste abrasivo, para aplicaciones de recargue. La información respecto de estos nuevos consumibles es escasa, en especial sobre el efecto que los parámetros de soldadura utilizados (calor aportado, gas de protección, voltaje de trabajo, intensidad de corriente, etc.) tienen sobre el depósito, su microestructura y por consiguiente su comportamiento frente al desgaste. Por tal motivo, en el presente trabajo se estudió el efecto del aporte térmico (tensión, corriente y velocidad de soldadura) sobre el depósito nanoestructurado base hierro, obtenido por FCAW sin protección gaseosa; este trabajo es continuación de otro en el que se estudió el mismo consumible soldado bajo protección gaseosa. Se soldaron muestras con aportes térmicos de entre 0,5 y $3,5 \mathrm{~kJ} / \mathrm{mm}$. Se realizó el relevamiento dimensional de los cordones, se analizó la composición química y la microestructura usando microscopías óptica y electrónica de barrido y difracción de rayos X. También se midieron la microdureza del depósito, el tamaño de cristalita y el grado de dilución. Se observó una gran influencia de las condiciones de proceso sobre la geometría del cordón y la microestructura. La operatividad de este consumible, soldado sin protección gaseosa fue muy buena.

Palabras clave: recubrimientos duros, alambre autoprotegido, nanomateriales, calor aportado, microestructura.

\begin{abstract}
Lately modern hardfacing tubular wires for semiautomatic welding with gas protection and without it have been developed. These wires deposit Fe-based nanostructured alloys with high abrasive wear resistance. The information on these new consumables is scarce, especially about the effect that the welding parameters (heat input, shielding gas, arc voltage, current intensity, etc.) have on the deposit, its microstructure and, consequently, its wear behavior. For this reason, in this work it was studied the effect of heat input (voltage, intensity of current and welding velocity) on the properties of a FCAW Fe-based nanostructured deposit obtained without gas protection. This study is a continuation of a previous one in which the same consumable was used under shielding gas. Bead on plate samples were welded using heat inputs between 0.5 and $3.5 \mathrm{~kJ} / \mathrm{mm}$. The dimensional study was performed on the beads, chemical composition was determined and the microstructure was analyzed using optical and electronic microscopy and X Ray diffraction. Microhardness, cristalita size and dilution percentage were also measured. It was observed an important influence of the welding parameters on the bead geometry and the microstructure. The operational behavior of this wire, welded without shielding gas, was very good.
\end{abstract}

Key words: hardfacing deposits, open arc wire, nanomaterials, heat input, microstructure.

\section{Introducción}

El proceso de soldadura semiautomático con alambre tubular (FCAW) es uno de los más utilizados para el recargue de pieza nuevas o recuperación de usadas. Es posible depositar capas con

Recebido em 11/06/2014, texto final em 13/11/2014.

DOI: http://dx.doi.org/10.1590/0104-9224/SI1904.04 gran eficiencia desde unos pocos hasta unas decenas de kilos de depósito de metal de soldadura por hora [1-2].

En los últimos años ha habido un gran interés en la producción de los alambres tubulares autoprotegidos que depositan aleaciones de hierro con una matriz de $\alpha$-ferrita nanoestructurada con carburos y boruros precipitados [3-5] lo que les otorga a los depósitos una alta resistencia al desgaste abrasivo y erosivo. A partir de recientes estudios y de la sensibilidad de estos alambres a los parámetros operativos, se decidió profundizar en el comportamiento de estos nuevos consumibles y estudiar la 
influencia del aporte térmico sobre la geometría del cordón, la dilución, la microestructura, el tamaño de cristalita y la microdureza de depósitos de aleaciones base Fe nanoestructuradas, obtenidos con un alambre tubular mediante el proceso de soldadura semiautomático sin protección gaseosa. Este trabajo es continuación de otro ya publicado [6], en el que se estudió el mismo consumible, soldado con el mismo proceso pero con protección gaseosa de $\mathrm{Ar}-20 \% \mathrm{CO}_{2}$.

\section{Materiales y métodos}

\subsection{Consumible utilizado}

El consumible empleado fue un alambre tubular comercial de diámetro 1,6 $\mathrm{mm}$, depositado mediante el proceso semiautomático de soldadura sin protección gaseosa, en forma mecanizada, empleando un dispositivo Miggytrac.

\subsection{Soldadura del cupón de aporte puro}

A fin de determinar la composición química del depósito libre de dilución, se soldó un cupón de aporte puro para análisis químico (figura 1). La secuencia del mismo fue de 3 capas con 4, 3 y 2 cordones en posición plana. Los parámetros de soldadura utilizados fueron de $300 \mathrm{~A}, 35 \mathrm{~V}$ y $5 \mathrm{~mm} / \mathrm{s}$ de velocidad de avance.

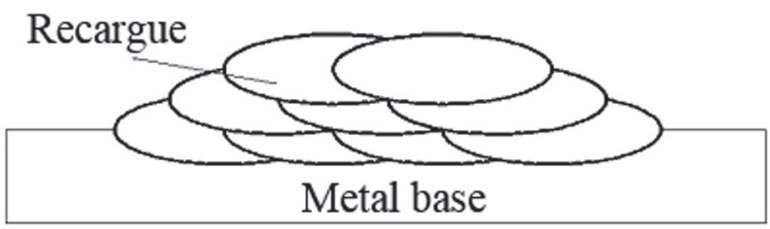

Figura 1. Esquema del cupón de aporte puro.

\subsection{Soldadura de las probetas para estudio del calor apor- tado (CA)}

Se soldaron 8 cupones en posición plana, con un cordón cada uno, sobre chapas base de $150 \times 75 \times 12,5 \mathrm{~mm}$ de acero al carbono tipo SAE 1010, como se muestra esquemáticamente en la figura
2. Los parámetros de soldadura empleados se pueden ver en la tabla 1, así como la identificación empleada y el calor aportado en cada caso. La longitud libre del alambre fue de $25 \mathrm{~mm}$ en todos los casos. Las distintas probetas se agruparon en dos series denominadas con las letras L (low=bajo) y H (high=alto), asociadas a la potencia eléctrica empleada; el número agregado a la letra corresponde a la velocidad de soldadura utilizada.

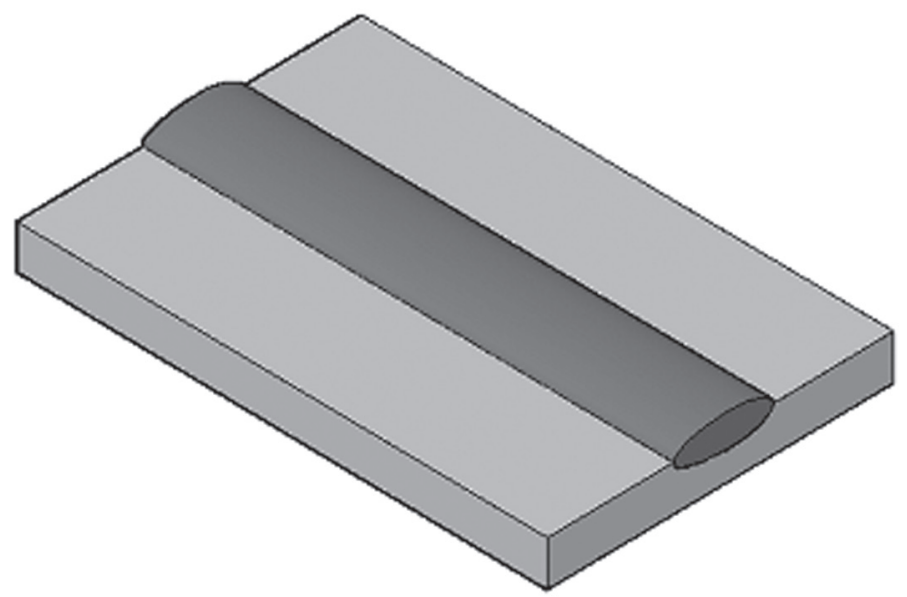

Figura 2. Esquema de la probeta de simple cordón.

De acuerdo con los parámetros de soldadura elegidos, dos pares de probetas fueron soldados con distintas potencia y velocidad de soldadura, pero con similar o igual calor aportado. Son las probetas $\mathrm{H} 7$ y L3 con 1,5 y 1,7 kJ/mm de calor aportado, respectivamente y, por otro lado, H10 y L5 ambas con $1,0 \mathrm{~kJ} / \mathrm{mm}$.

Luego de soldadas las distintas probetas se realizó una inspección visual a fin de detectar la presencia de defectos macroscópicos superficiales.

\subsection{Caracterizaciones química y macro y microestructura- les de las probetas $\mathrm{CA}$}

Sobre los cortes transversales se realizaron mediciones de composición química mediante espectrometría de energía dispersiva de rayos X (EDS). Las dimensiones de los cordones - ancho, penetración y sobremonta - se midieron mediante software de análisis de imágenes. Se caracterizó la microestructura mediante microscopía electrónica de barrido (SEM).

Tabla 1. Parámetros de soldadura.

\begin{tabular}{|c|c|c|c|c|c|}
\hline Identificación & Tensión de arco $(V)$ & $\begin{array}{c}\text { Intensidad de } \\
\text { corriente }(A)\end{array}$ & $\begin{array}{c}\text { Potencia eléctrica } \\
(\mathrm{kW})\end{array}$ & $\begin{array}{c}\text { Velocidad de } \\
\text { soldadura }(\mathrm{mm} / \mathrm{s})\end{array}$ & $\begin{array}{c}\text { Calor aportado } \\
(\mathrm{kJ} / \mathrm{mm})\end{array}$ \\
\hline L3 & 25 & 200 & 5,0 & 3 & 1,7 \\
\hline L5 & 25 & 200 & 5,0 & 5 & 1,0 \\
\hline L7 & 25 & 200 & 5,0 & 7 & 0,7 \\
\hline L10 & 25 & 200 & 5,0 & 3 & 0,5 \\
\hline H3 & 35 & 300 & 10,5 & 5 & 3,5 \\
\hline H5 & 35 & 300 & 10,5 & 7 & 1,5 \\
\hline H7 & 35 & 300 & 10,5 & 10 & 1,0 \\
\hline H10 & 35 & 300 & 10,5 & & \\
\hline
\end{tabular}

L: baja potencia elétrica; $\mathbf{H}$ : alta potencia elétrica; 
Sobre la superficie de cada cordón se realizó difracción de rayos X (DRX). El equipo empleado fue un RIGAKU, con radiación de $\mathrm{Cu} \mathrm{K}-\alpha$, entre $35^{\circ}$ y $95^{\circ}$, con una velocidad de barrido de $1 \%$ min. A partir de los espectros obtenidos se analizaron las fases presentes y se determinó el tamaño de cristalita de la fase $\alpha$-ferrita, utilizando la fórmula de Scherrer [7-8].

\subsection{Estudio de dilución de las probetas CA}

El porcentaje de dilución fue calculado a partir de las relaciones de áreas entre el metal fundido y el metal base. Las mediciones se realizaron a través de un software de análisis de imágenes. También se efectuaron estimaciones de dilución a través de variaciones del $\%$ de $\mathrm{Cr}$ entre la chapa base y el metal de aporte puro.

\subsection{Estudio de dureza sobre las probetas CA}

Sobre los cortes transversales se midió la microdureza Vickers (HV2) a $1 \mathrm{~mm}$ de la superficie, en cinco zonas, promediándose los valores obtenidos. También se determinó la microdureza sobre las fases con HV0,05.

\section{Resultados y discusión}

\subsection{Composición química del metal de aporte puro}

En la tabla 2 se muestra el resultado del análisis químico obtenido de la probeta de metal de aporte puro.
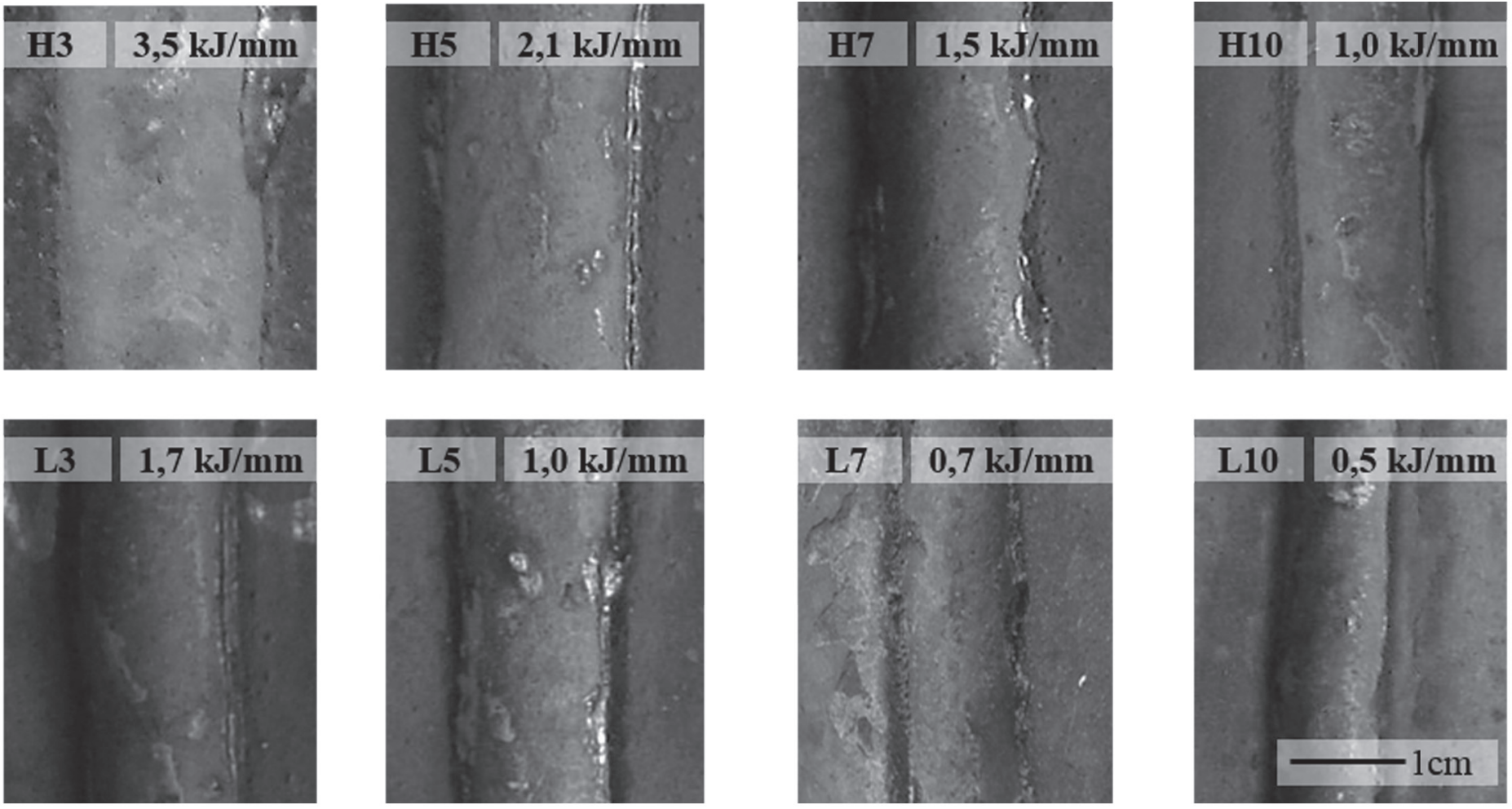

El material depositado presentó una alta concentración de elementos de aleación, dentro del sistema Fe-(Nb,Cr)-(C,B). La composición química cumple con las reglas para la formación de nanoestructuras, las cuales son: a) que el sistema sea multicomponente, con al menos tres elementos de aleación; b) una diferencia de radio atómico entre los elementos que constituyen el sistema de aleación mayor del $12 \%$ y c) que el calor de mezcla entre sus tres elementos principales sea negativo [9-10]. Estas características producen grandes dificultades para la nucleación y el crecimiento de capas de átomos regulares, produciendo cristales de tamaño nanométrico.

\subsection{Análisis de las probetas $\mathrm{CA}$}

\subsubsection{Inspección visual}

Las fotos adquiridas de los diferentes cordones soldados se muestran en la figura 3.

Se encontró escoria sobre los laterales de los cordones. Las salpicaduras fueron pocas y de pequeño tamaño. La terminación superficial fue buena, no se observaron líneas de playa. Se produjeron fisuras transversales al cordón producidas por el alivio de tensiones, típicas en estos materiales.

Figura 3. Vista superior de los cordones soldados (1x). 

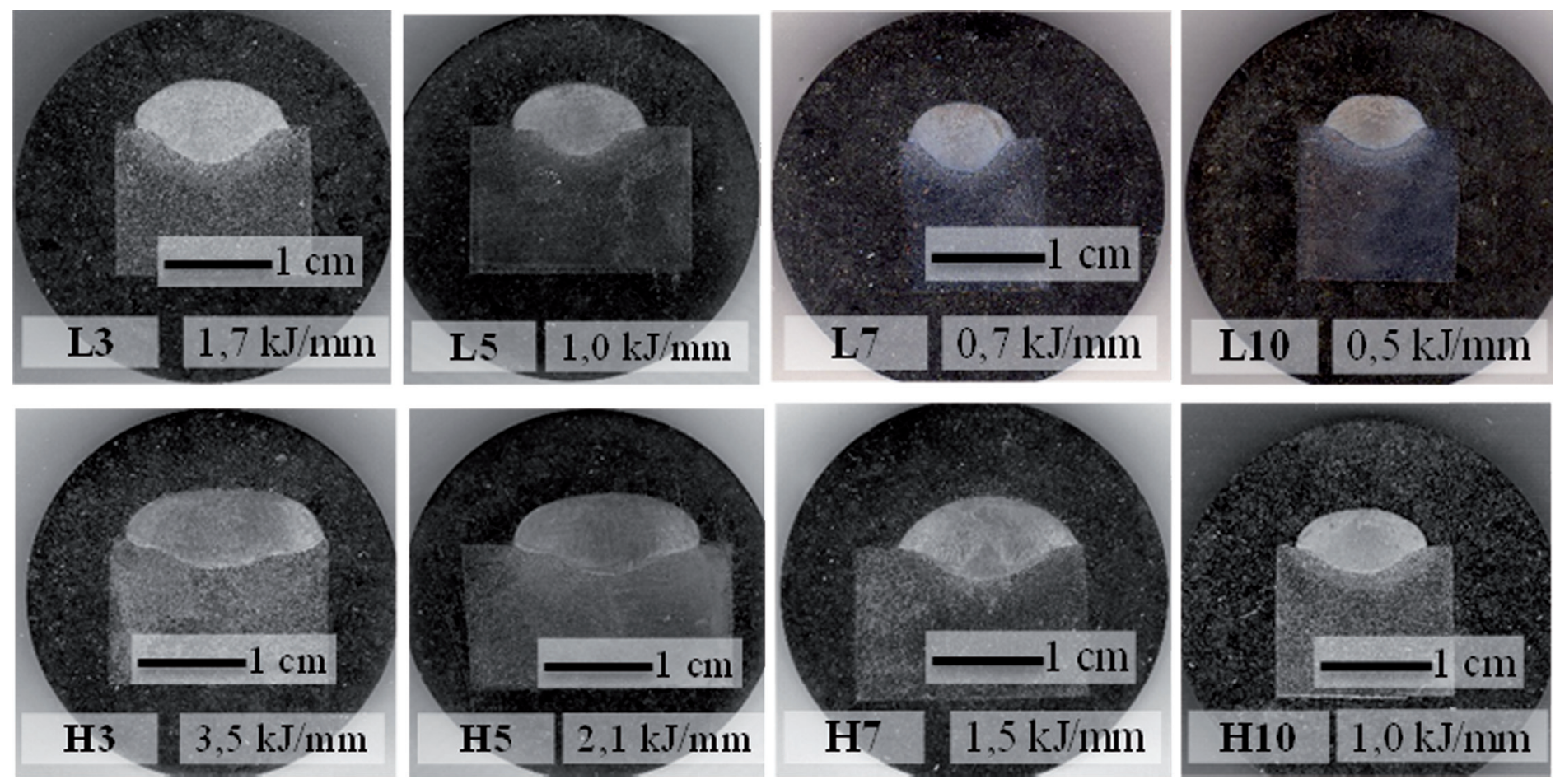

Figura 4. Macroestructura de las probetas soldadas sin protección gaseosa.

Tabla 3. Ancho, penetración y sobremonta medidos sobre los distintos cordones depositados.

\begin{tabular}{|c|c|c|c|c|c|c|c|c|}
\hline Probeta & L3 & L5 & $\boldsymbol{L 7}$ & $\boldsymbol{L 1 0}$ & $\boldsymbol{H 3}$ & $\boldsymbol{H 5}$ & $\boldsymbol{H 7}$ & $\boldsymbol{H 1 0}$ \\
\hline Calor aportado $(\mathrm{kJ} / \mathrm{mm})$ & 1,7 & 1,0 & 0,7 & 0,5 & 3,5 & 2,1 & 1,5 & 1,0 \\
\hline Velocidad de avance $(\mathrm{mm} / \mathrm{s})$ & 3 & 5 & 7 & 10 & 3 & 5 & 7 & 10 \\
\hline Ancho de cordón $(\mathrm{mm})$ & 12,4 & 11,0 & 8,4 & 8,0 & 16,6 & 15,5 & 14,6 & 10,3 \\
\hline Penetración $(\mathrm{mm})$ & 3,0 & 2,5 & 2,3 & 1,8 & 2,8 & 2,5 & 2,4 & 2,1 \\
\hline Sobremonta $(\mathrm{mm})$ & 3,8 & 3,6 & 3,4 & 2,7 & 4,2 & 4,2 & 4,1 & 3,4 \\
\hline
\end{tabular}

\subsubsection{Macrografía y análisis dimensional de los cordones}

No se observaron defectos macroscópicos, como poros o inclusiones: figura 4 . Se encontró buena adherencia de los cordones; éstos presentaron buen mojado.

Se ob ervó que la forma de la línea de fusión en las probetas soldadas (L3, H3, L5 y H5) fue en forma de "hongo".

Los valores medidos de ancho, penetración y sobremonta de cada uno de los cordones se presentan en la tabla 3.

En la figura 5 se grafican los cambios del ancho, la penetración y la sobremonta del cordón con la velocidad de soldadura, con cada serie L y H por separado, a fin de ver el efecto de la potencia empleada.

Como tendencia general, con el aumento de la velocidad de soldadura (o disminución del calor aportado) se produjo una disminución en los valores de ancho de cordón (de 16 a $8 \mathrm{~mm}$ ), penetración (de $3 \mathrm{~mm}$ a 1,8 $\mathrm{mm}$ ) y sobremonta (de 4 a $2 \mathrm{~mm}$ ). El incremento de la potencia eléctrica aplicada generó aumentos en el ancho de cordón y la sobremonta, no pareciendo haber tenido importante influencia sobre la penetración. Esto es consistente con lo reportado en la bibliografía [2]. Las probetas con similar calor aportado presentaron variaciones en la geometría de los cordones, principalmente en el ancho. Esto se explicaría por el aumento de tensión del arco que tiende a aplanar al cordón y la mayor deposición con el aumento de la corriente [2].

Es interesante remarcar que al aumentar la potencia eléctrica (para los mismos valores de calor aportado), se observaron mayores sobremonta y ancho y poca variación en la penetración, todas características deseables para el recargue.

\subsubsection{Estudios de dilución}

Los resultados de los cálculos de dilución [2], realizados en base a las fotos macro de los cortes transversales, se presentan en la tabla 4. 

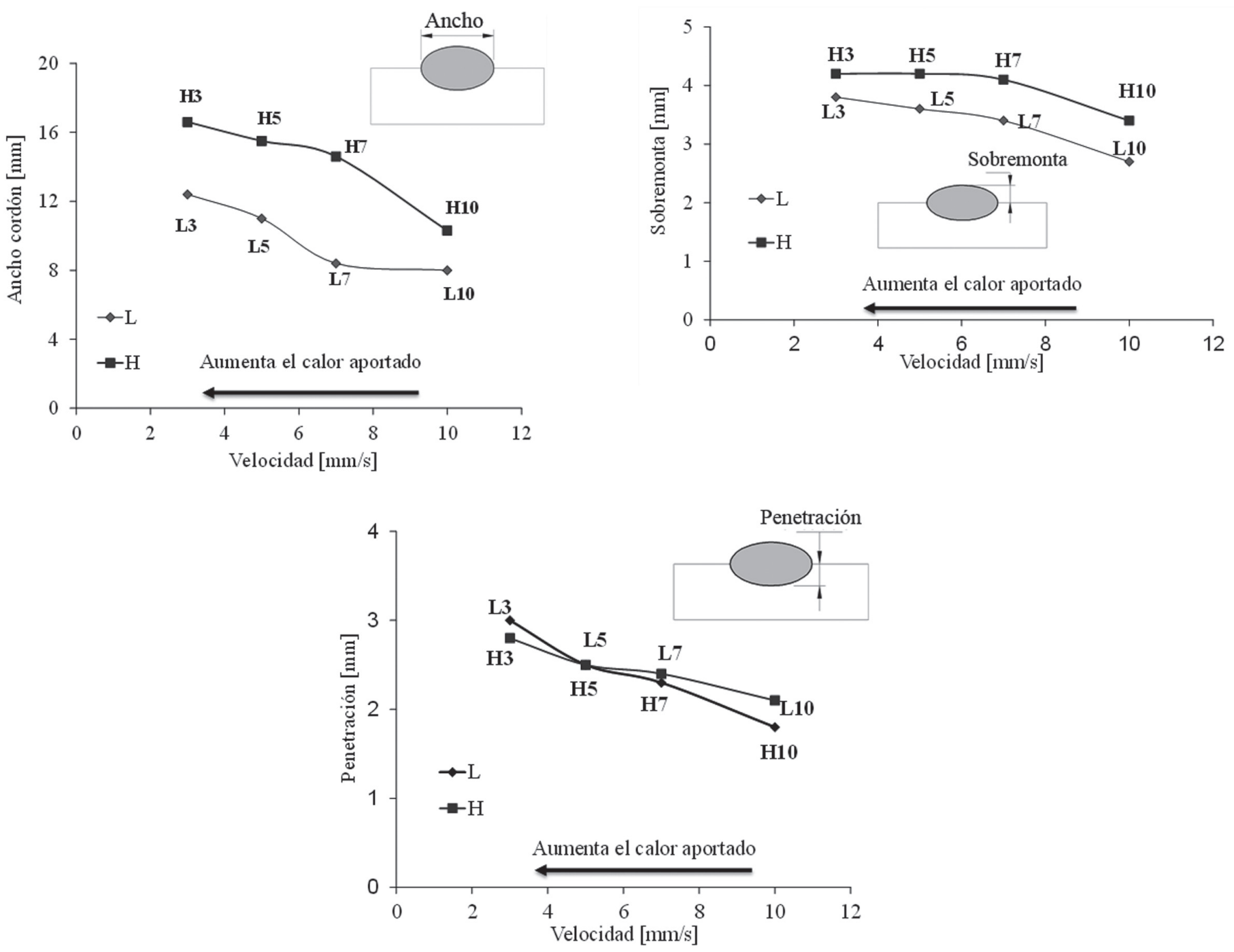

Figura 5. Ancho de cordón, sobremonta y penetración vs. velocidad de soldadura.

Tabla 4. Dilución para las distintas condiciones de soldadura.

\begin{tabular}{|c|c|c|c|c|c|c|c|c|}
\hline Probeta & L3 & L5 & L7 & L10 & H3 & H5 & H7 & H10 \\
\hline Calor aportado $[\mathrm{kJ} / \mathrm{mm}]$ & 1,7 & 1,0 & 0,7 & 0,5 & 3,5 & 2,1 & 1,5 & 1,0 \\
\hline Dilución (\%) & 32 & 28 & 37 & 38 & 28 & 25 & 32 & 33 \\
\hline
\end{tabular}

Tabla 5. Concentración de $\mathrm{Cr}$ en los distintos cordones depositados (\% en peso).

\begin{tabular}{|c|c|c|c|c|c|c|c|c|}
\hline Probeta & $L 3$ & $L 5$ & $L 7$ & $L 10$ & $H 3$ & $H 5$ & $H 7$ & $H 10$ \\
\hline Calor aportado $[\mathrm{kJ} / \mathrm{mm}]$ & 1,7 & 1,0 & 0,7 & 0,5 & 3,5 & 2,1 & 1,5 & 1,0 \\
\hline $\mathrm{Cr}$ & 10,2 & 10,9 & 9,8 & 10,3 & 12,4 & 12,7 & 11,5 & 11,6 \\
\hline
\end{tabular}

El porcentaje de dilución varió entre 25 y $38 \%$ para las distintas condiciones de soldadura. Se observó que los menores valores de dilución se presentaron en las probetas soldadas con alto calor aportado. Esto estaría asociado con el mayor aporte de material que se produce en la soldadura sin protección gaseosa con el aumento de la potencia eléctrica, y con la forma de la línea de fusión que genera una baja mezcla con el metal base (figura 4).

Los resultados obtenidos de los valores de $\mathrm{Cr}$ se presentan en la tabla 5 .

Se observó que al aumentar el aporte térmico hubo una mayor concentración de Cr. Esto podría explicarse por la menor dilución debido al mayor aporte de material y a una baja penetración [2]. No se observaron grandes diferencias respecto de la variación de la velocidad.

A partir de los datos obtenidos de la composición del metal del metal de aporte puro y del material base para cada cordón se calcularon los valores promedio de dilución de cada probeta. En la figura 6 se muestran los resultados obtenidos junto con los valores de dilución determinados en la tabla 3 . 


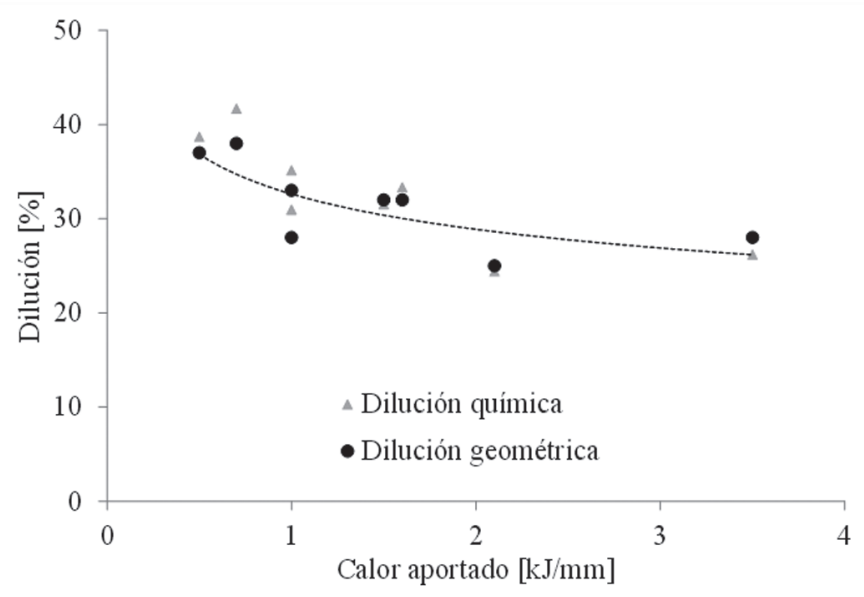

Figura 6. Relación entre las diluciones química y geométrica y el calor aportado de todos los cordones.

Se observaron diferencias menores al 10\% entre la dilución medida sobre las macros y los cálculos realizados considerando la composición química. Esto estaría asociado a la oxidación producida durante las reacciones en la pileta líquida.
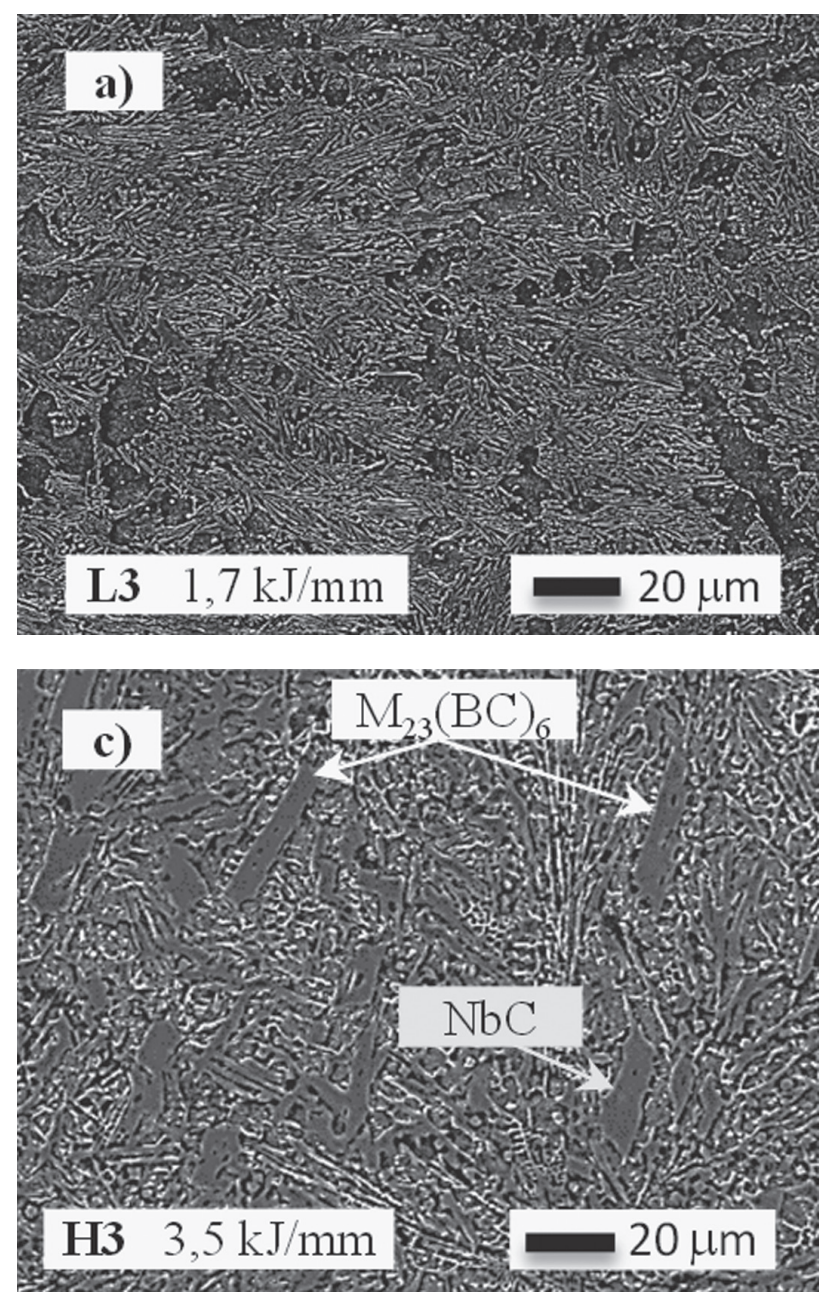

En contraste con lo reportado en el trabajo previo [6] del que éste es continuación, donde se soldó bajo gas Ar- $20 \% \mathrm{CO}_{2}$, se puede ver que al aumentar el calor aportado disminuyó la dilución. Esto es consistente con lo observado en los gráficos de penetración, ancho y sobremonta y en los cortes macro. Ésta es la condición ideal: se obtiene máximo aporte y baja dilución.

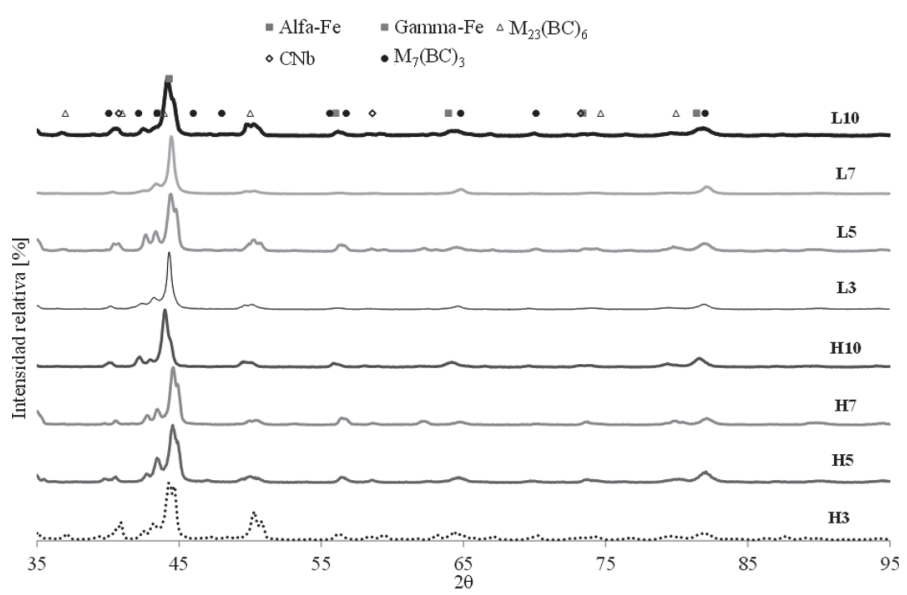

Figura 7. Espectros de XRD de todas las condiciones.
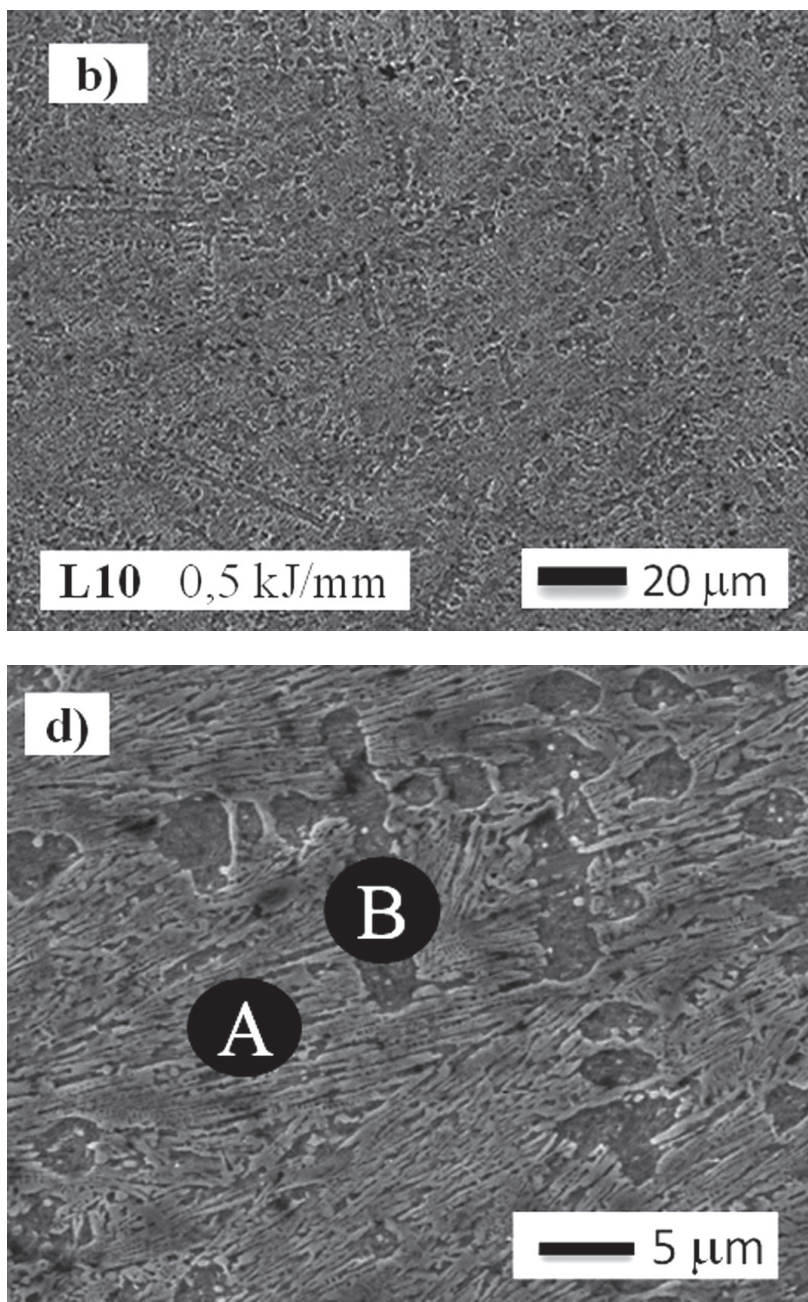

Figura 8. Micrografías SEM de las probetas L10, L3 y H3. 


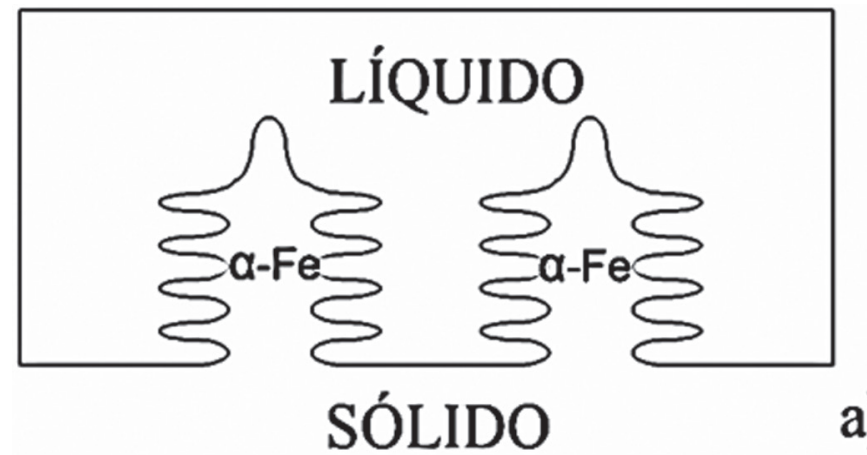

a)

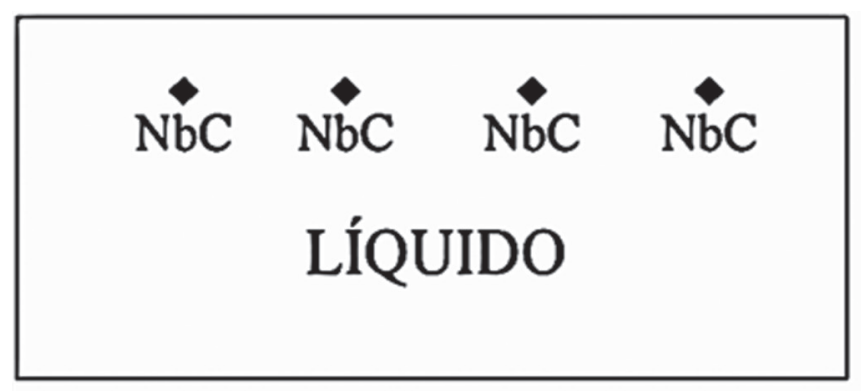

\section{SÓLIDO}

c)

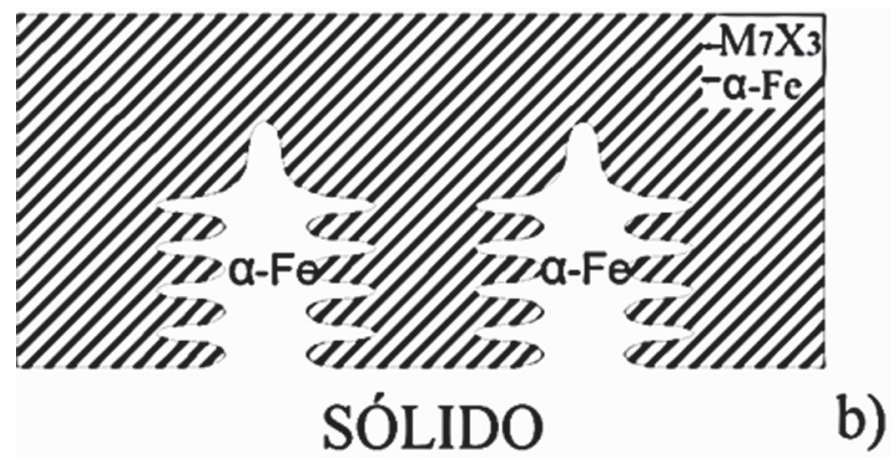

b)

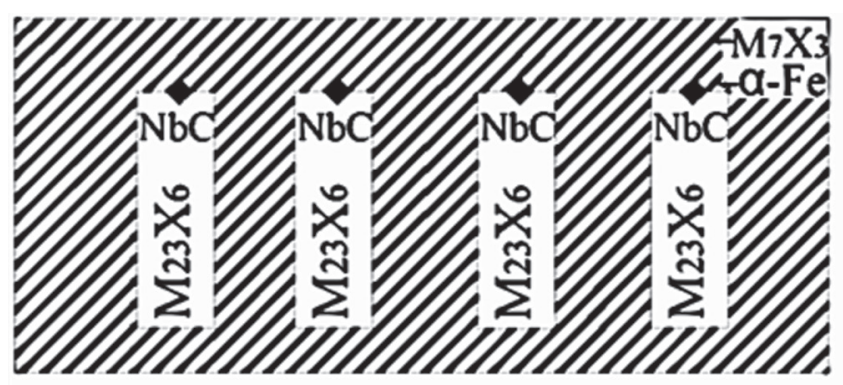

SÓLIDO

d)

Figura 9. Modos de solidificación.

\subsubsection{Caracterización microestructural}

Sobre la superficie de todas la muestras se realizaron espectros de DRX, figura 7.

Se puede ver que la estructura estuvo formada principalmente por $\alpha$-Fe, carboboruros metálicos $\left(\mathrm{M}_{7}(\mathrm{BC})_{3}, \mathrm{M}_{23}(\mathrm{BC})_{6}\right)$ y carburos de niobio $(\mathrm{NbC})$. Se observaron variaciones de las fases presentes con el aporte térmico para las probetas soldadas con alto calor aportado H3 y H5 (menor dilución) que mostraron mayor presencia de $\mathrm{M}_{23} \mathrm{C}_{6}$. Esto estaría relacionado con el mayor contenido de elementos de aleación producto de la menor dilución que se obtuvo en estas últimas probetas. El tamaño de cristalita fue de 105 y $125 \mathrm{~nm}$, variaciones que podrían estar relacionadas con el porcentaje total de elementos de aleación en la matriz [11-12], lo que podría afectar la formación de los nanocristales.

En la figura 8 se muestran las imágenes SEM de las microestructuras.

En la figuras $8 \mathrm{a}$ y $8 \mathrm{~b}$ (L3 y L10) se puede observar un patrón de segregación dendrítico, donde los valores de $\mathrm{Cr}$ fueron de $12 \%$ en la zona interdendrítica y $7 \%$ dentro de la región dendrítica, indicaciones A y B en la figura 8d, respectivamente. Dicho patrón se volvió más fino a medida que disminuyó el aporte térmico. En la zona interdendrítica se ve una estructura laminar/globular $\left(\mathrm{M}_{7} \mathrm{X}_{3}\right)$, que también se afinó al disminuir el aporte térmico.

La figura 8c muestra la presencia de $\mathrm{M}_{23}(\mathrm{CB})_{6}$ y $\mathrm{NbC}$.

En función del calor aportado y la dilución, se observaron 2 patrones de solidificación.

En el primero se forma la fase $\alpha-\mathrm{Fe}$ y a continuación, en la zona interdendrítica precipita el eutéctico, constituido por láminas planas y globulares de precipitados del tipo $\mathrm{M}_{7} \mathrm{X}_{3}$ y $\alpha-\mathrm{Fe}$ (figuras 9a y 9b). En el segundo, encontrado en las muestras con baja dilución (H3 y H5), precipitan inicialmente los $\mathrm{NbC}, \mathrm{y}$ posteriormente, con la disminución de la temperatura, nuclean sobre ellos los carboboruros complejos $\mathrm{M}_{23} \mathrm{X}_{6}$, como se indica en la figura 9c; finalmente, a partir del líquido remanente se forma el eutéctico, $M_{7} X_{3}$ y $\alpha-F e$ [13-15], figura 9d. Este modo de solidificación fue encontrado en trabajos recientes $[16,17]$ con el consumible en estudio, realizados en 1 y 2 capas, donde la dilución fue más baja.

\subsubsection{Microdureza}

En la tabla 6 se observan los resultados de la microdureza medidos, promedio de por lo menos tres mediciones.

Puede verse que los valores variaron entre 825 y 882 HV2, siendo consistentes con lo esperado para este material $[15,18$ -

Tabla 6. Microdureza [HV2].

\begin{tabular}{|c|c|c|c|c|c|c|c|c|}
\hline & L3 & L5 & L7 & L10 & H3 & H5 & H7 & H10 \\
\hline Probeta & 1,7 & 1,0 & 0,7 & 0,5 & 3,5 & 2,1 & 1,5 & 1,0 \\
\hline Calor aportado $[\mathrm{kJ} / \mathrm{mm}]$ & 32 & 28 & 37 & 38 & 28 & 25 & 32 & 33 \\
\hline Dilución (\%) & 867 & 882 & 871 & 856 & 833 & 837 & 829 & 825 \\
\hline Dureza &
\end{tabular}



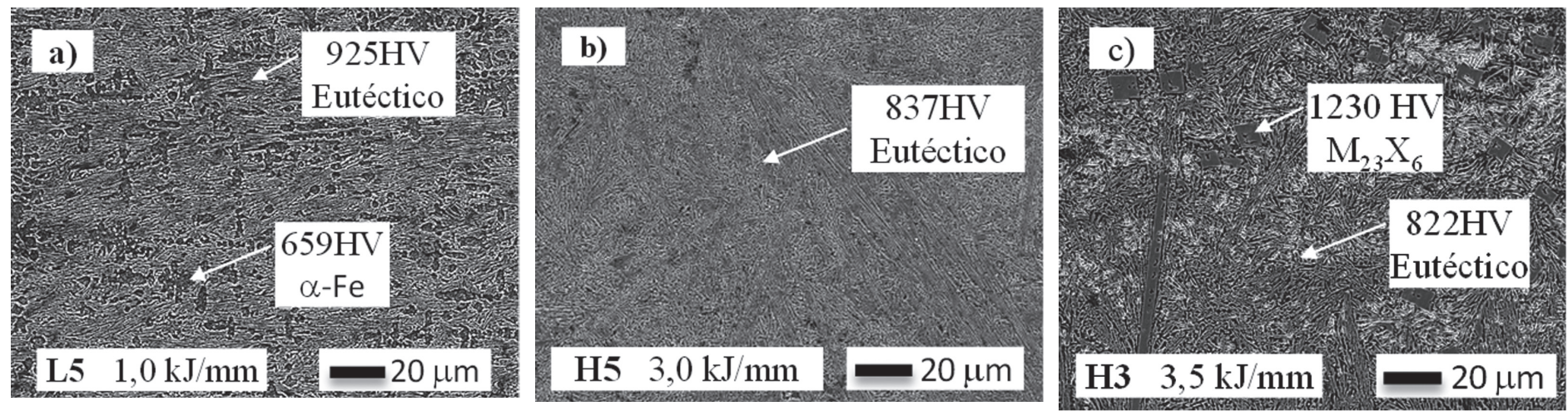

Figura 10. Valores de microdureza de las fases de las probetas L5, H5 y H3. Eutéctico: $\alpha-\mathrm{Fe}+\mathrm{M}_{7}(\mathrm{BC})_{3}$.

19]. Las probetas con similar aporte térmico presentaron variaciones en la dureza a pesar de tener casi la misma dilución, lo que estaría relacionado con las fases presentes y su microdureza.

Se observó que la microdureza de la fase eutéctica aumentó con el aumento de la velocidad de soldadura. Esto estaría asociado a un afinamiento de dicha fase lo que produciría una mayor restricción del movimiento de las dislocaciones y en consecuencia mayor dureza [20]. También se observó una disminución de la fracción de fase $\alpha$-Fe con el aumento del calor aportado (L5 mayor fracción de $\alpha$-Fe que H5 y ésta mayor que H3), figura $10 \mathrm{a}, 10 \mathrm{~b}$ y $10 \mathrm{c}$, y un eutéctico globular/laminar más grueso de menor microdureza $(925,837,822 \mathrm{Hv}$ para L5, H5 y H3 respectivamente). Este fenómeno se explicaría por la menor dilución, figura 6 , lo que favorece el segundo modo de solidificación. También se observó la presencia de caboboruros $\mathrm{M}_{23} \mathrm{X}_{6}$ de gran dureza, que probablemente tuvieron una baja incidencia en la dureza macro debido a su baja fracción en volumen, evaluada visualmente.

\section{Conclusiones}

En el presente trabajo se evaluó el efecto de los parámetros de soldadura sobre las características de los depósitos de una aleación nanoestructurada base $\mathrm{Fe}$, obtenidos con un alambre tubular por el proceso de soldadura semiautomático, sin protección gaseosa, con aportes térmicos entre 0,5 y $3,5 \mathrm{~kJ} / \mathrm{mm}$.

\section{Se concluyó que:}

- Todas las probetas soldadas presentaron una buena terminación superficial, bajos niveles de salpicaduras y de escorias. La mayoría de ellas fisuraron durante el enfriamiento.

- A mayor velocidad de soldadura (o disminución del calor aportado) se produjo una disminución en los valores de ancho de cordón, penetración y sobremonta. El incremento de la potencia eléctrica aplicada generó aumentos en el ancho de cordón y la sobremonta, no pareciendo haber tenido importante influencia sobre la penetración. Las probetas soldadas con similar calor aportado presentaron variaciones en la geometría de los cordones, principalmente en el ancho.

- El material depositado presentó una dilución de $25-28 \%$ para las probetas soldadas con los mayores aportes térmicos y de 33$38 \%$ para aquéllas soldadas con los menores aportes térmicos.

- La microestructura estuvo formada por una matriz de $\alpha-F e$, carboboruros $\left(\mathrm{M}_{7}(\mathrm{BC})_{3}, \mathrm{M}_{23}(\mathrm{BC})_{6}\right)$, y $\mathrm{NbC}$. Se observaron variaciones de las fases presentes con el aporte térmico: $\mathrm{H3}$ y $\mathrm{H} 5$ (menor dilución) mostraron mayor presencia de $\mathrm{M}_{23} \mathrm{C}_{6}$. El tamaño de cristalita, varió de 105 y $125 \mathrm{~nm}$. Se observó un patrón de segregación dendrítico más fino con menor aporte térmico. En la zona interdendrítica se vio una estructura laminar/globular con el mismo comportamiento. Se observó que la fracción bifásica aumentó al aumentar el calor aportado.

- La dureza varió con el aporte térmico desde 825 hasta 882 HV. Las probetas con similar aporte térmico presentaron variaciones en la dureza a pesar de tener casi la misma dilución. Se observó que la microdureza de la fase eutéctica aumentó con el aumento de la velocidad de soldadura. También se observó una disminución de la fracción de la fase $\alpha$-Fe con el aumento del calor aportado y un eutéctico globular/laminar más grueso de menor microdureza. Se detectó la presencia de caboboruros $\mathrm{M}_{23} \mathrm{X}_{6}$ de gran dureza.

- En conclusión, los resultados presentados muestran que es posible soldar con alto calor aportado, obteniéndose baja dilución del material de recargue, lo que favorece el segundo modo de solidificación, que genera mayor proporción de carburos duros, lo que es deseable en estas aplicaciones.

\section{Agradecimientos}

Los autores agradecen a EUTECTIC-CONARCO Argentina por la provisión del consumible utilizado, a AIR LIQUIDE Argentina por la donación de los gases de soldadura, a EUTECTIC-USA por la realización de los análisis químicos, al LABORATORIO DE MICROSCOPÍA ELECTRÓNICA DE INTI MECÁNICA por la realización de la microscopía electrónica de barrido y a APUENFI (Asociación de Profesores Empresarios Área Mecánica de la Facultad de Ingeniería-Universidad Nacional de Lomas de Zamora) por el apoyo económico al presente proyecto.

\section{Referencias bibliográficas}

[1] LINNERT, G.E., Welding metallurgy of carbon and alloy steels. 4 ed. AWS: Miami, Florida. 1994. 474 p.

[2] MERRICK, S.; KOTECKI, D.; WU, J., Materials and applications - Part 2. Welding Handbook, American Welding Society. 1998. 
[3] HEATH, G., Nanotechnology and Welding - Actual and possible future applications. Proceedings of the CASTOLIN-EUTECTIC SEMINAR, Brussels: Belgium, 2006. p. 25-35.

[4] ALLEN, S; EDWIN, T., The Structure of Materials. New York: John Wiley \& Sons, Inc. 1999.

[5] KLIMPEL, A; JANICKI, D., A study of worn wear plates of fan blades of steel mill fumes suction system, Proceedings of the 13th Scientific International Conference, "Achievements in Mechanical and Materials Engineering" AMME'2005, Polonia: Gliwice, 2005. p. 307-310.

[6] GUALCO, A.; SVOBODA, H.G.; SURIAN, E.S. Efecto de los parámetros de soldadura sobre la microestructura de recargues nanoestructurados base hierro". A. Gualco, H. G. Svoboda. E. Surian, Soldagen \& Inspecao, vol 18, nº , 2013.

[7] CULLITY, B.D.; STOCK, S.R. Elements of X-ray diffraction. 3 ed. Prentice Hall. 2001. 520 p

[8] Software de análisis de espectros de difracción Xpowder, versión 2004.

[9] Datasheet: TeroMatec 395NOA, Eutectic Castolin-ESAB, USA, 2008.

[10] GLEITER, H., Nanostructured materials: basic concepts and microstructure. Acta Materialia, v.48, n.1, p.1-29. 2000.

[11] WEISSMIILLER J. Alloy effects in nanostructures. Nanostructured Materials, v. 3, p.261-272. 1993.

[12] INOE, A. Amorphous and nanocrystalline materials: Preparation, properties, and applications. Springer, p.206, 2010.

[13] ZHI, X.; XING, J.; FU, H., "Effect of niobium on the ascast microstructure of hypereutectic high chromium cast iron"; Materials Letters 62, p 857-860, 2008.

[14] MAA, A; XINGA, J.; LIUA, S; Li, Y. "Effect of chromium concentration on microstructure and properties of $\mathrm{Fe}-3.5 \mathrm{~B}$ alloy"; Materials Science and Engineering A, A 527, p. 68006808, 2010 .

[15] BRANAGAN, D.J.; MARSHALL, M.C.; Meacham, B.E., High toughness high hardness iron based PTAW weld materials. Materials Science and Engineering A 428, p. 116-123, 2006.

[16] GUALCO, A.; SVOBODA, H.G.; SURIAN, E.S. Efecto del número de capas de soldadura sobre la resistencia al desgaste de recargues nanoestructurados base $\mathrm{Fe}$, soldados con protección gaseosa y sin ella, XL Consolda, Sao Paulo, Brasil, 20-23, 0ctubre, 2014.

[17] GUALCO, A.; MARINI, C.; SVOBODA, H.G.; SURIAN, E.S., Efecto del tratamiento térmico post-soldadura sobre la resistencia al desgaste de recargues nanoestructurados base hierro. SAM/CONAMET, 20-23 de octubre 2014, Santa Fe, Argentina. [18] WEISSMIILLER J. Some basic notions on nanostructured solids. Materials Science and Engineering, A v.179-180, p.102107. 1994.

[19] MORRIS D.G. The origins of strengthening in nanostructured metals and alloys. Revista de Metalurgia, v.46, n.2, p.173186. 2010.

[20] VERHOEVEN J.D. Fundamentos de la Metalúrgia Física. Ed Limusa, 1er versión, 1987. 